The Journal of the Astronautical Sciences, Vol. 57, Nos. 1 \& 2, January-June 2010, pp. 73-92

\title{
Generalized Wahba Problems for Spinning Spacecraft Attitude and Rate Determination ${ }^{1}$
}

\author{
Mark L. Psiaki ${ }^{2}$
}

\begin{abstract}
Two generalized versions of Wahba's attitude determination problem have been developed for a spinning spacecraft, and a restricted version of one problem has been solved in closed-form. These problems seek to estimate both attitude and rate based solely on a time series of vector attitude observations along with a spacecraft dynamic model. Algorithms that solve these problems will be useful for spin-stabilized spacecraft that reduce complexity by omitting rate gyros. The first generalized Wahba problem presumes that the spin axis is known and that the spin rate is constant but unknown, as for a spinning spacecraft that has a nutation damper. The second generalized problem includes full rigid-body Euler dynamics, which allow for nutations, and seeks to estimate the unknown initial attitude rate vector. Both problems are recast into the $K$-matrix form of Wahba's problem with $K$ matrices that depend on the unknown rates. Restricted problems are developed that use the minimum number of vector measurements, two for the first problem and three for the second problem. The restricted first problem is solved in closed-form. The restricted second problem is shown to be observable, and it is reduced to a small system of nonlinear equations in the axially symmetric case. The possibility of deriving global solutions for these problems makes them attractive to assist or replace an extended Kalman filter because a global solution cannot suffer from nonlinear divergence.
\end{abstract}

\section{Introduction}

Attitude estimation for a spinning spacecraft is important because spinning spacecraft designs are often chosen for missions with a modest budget. A spinning spacecraft's attitude determination and control system tends to be more robust and less costly due to the gyroscopic stiffness afforded by its nonzero angular momentum, which makes the spacecraft's dynamic motions more predictable. In order to realize low cost, it is usually necessary to dispense with rate gyros. Such designs 
exploit the improved fidelity of the attitude rate dynamics model in order to enable rate estimation based solely on attitude sensor data.

This design approach results in a combined attitude and rate determination problem that is highly nonlinear. It can be solved using extended Kalman filter (EKF) methods, as in references [1] and [2]. Extended Kalman filters, however, can diverge or produce poor estimation accuracy if their linearization assumptions break down $[3,4]$.

Another important tool in the field of attitude estimation is Wahba's problem [5] and the $q$-method solution to this problem $[3,6]$. Wahba defined a nonlinear attitude determination problem, and the $q$ method solves it exactly in closed-form. There are no linearization assumptions in this approach, and, therefore, no linearization-induced divergence problems or accuracy reductions.

The original Wahba problem and $q$-method solution are limited to batch attitude determination from vector observations at a single instant of time. This original problem does not admit the possibility of estimating attitude rate along with attitude based on a time series of vector measurements. There have been attempts to extend the Wahba/q-method approach to a filtered form that also involves rate parameter estimates or rate measurements along with dynamic propagation [7-11]. All such attempts rely on rate gyros and a purely kinematic model for dynamic propagation. Either they do not estimate rate parameters $[9,10]$, or they estimate only rate-gyro biases and lose any guarantee of attaining a global solution $[7,8,11]$.

The present paper seeks to generalize Wahba's problem and to work towards generalizing $q$-method-like solutions in ways that encompass both attitude and rate estimation for a spinning spacecraft based only on vector observations. The generalized problems exploit knowledge of the attitude dynamics in order to eliminate the need for rate gyros. Solution algorithms are sought that simultaneously determine the attitude and rate estimates that constitute the global optimum of the generalized problem. These generalized problems include the possibility of measuring only one vector at any one instant in time. Otherwise, the problem becomes too easy because a $q$-method solution to Wahba's problem can be applied at each instant of time for which two or more vector observations are available. As much as is possible, this paper seeks to exploit existing knowledge about the $q$-method solution to Wahba's original problem because of its known ability to yield the global optimum. Note that the present generalization of Wahba's problem is different from the generalization of reference [12], which seeks only to add another measurement type to the original static problem.

The new algorithms will be useful for spin-stabilized spacecraft that use vector attitude observations from Sunsensors, magnetometers, or star cameras. In the case of star cameras, the new methods will be important only for an instrument with a very narrow field of view, i.e., one that often cannot return multiple vector measurements at a single point in time that have enough linear independence to yield an accurate $q$-method solution. Although they could be used alone, the new algorithms also might be useful for initializing the state of an EKF like one of those in reference [2]. They could ensure that the EKF did not diverge by providing a guaranteed level of initial state accuracy.

The present paper makes four main contributions to the problem of attitude and attitude-rate estimation for a spinning spacecraft. Its first contribution is to pose two generalizations of Wahba's problem for a spinning spacecraft. Both generalized problems involve solutions for three-axis attitude and rate based purely on a 
time series of reference vector observations, with the likely scenario of having only a single vector observation at each sample time. Both generalized problems are based on a torque-free Euler rigid-body dynamics model of a spinning spacecraft. The first problem presumes spin about a known body axis at an unknown constant spin rate. This problem is applicable to a spin-stabilized spacecraft with a nutation damper. The second generalized problem presumes that the spacecraft moments and products of inertia are known and that the unknown angular momentum is constant in inertial coordinates. This latter problem is applicable to a spinning/nutating spacecraft. Two forms of the second problem are discussed, the general form and a form that assumes axial symmetry.

The paper's second contribution is a closed-form solution of a restricted version of the first problem. The restricted problem includes only two vector measurements at two distinct sample times. This is the minimum number of measurements required in order to determine the unknown initial attitude and the unknown constant spin rate.

The third contribution is to pose a restricted form of the second problem: the unknown initial three-axis attitude and three-dimensional angular rate vector must be determined based on three vector measurements. This problem is not solved, but the observability of example systems is demonstrated based on only three vector measurements. Possible analytic solution strategies are discussed for this problem in the case of axial symmetry.

The final contribution of this paper is to outline possible solution strategies for the unrestricted forms of the two generalized Wahba problems. This discussion aims to encourage further research efforts that seek solutions to these problems.

The remainder of this paper consists of four main sections plus conclusions and an appendix. The second section reviews the original Wahba problem and the $q$ method solution, and it presents the two new generalized Wahba problems for a spinning spacecraft. The third section defines the restricted version of the first generalized Wahba problem. This section also develops an analytic solution to this restricted problem. The fourth section defines the restricted version of the second generalized Wahba problem, it demonstrates the local observability of this problem by using a gradient-based analysis, and it suggests how analytic solutions might be developed for the axially symmetric case. The fifth section discusses possible solution strategies for the unrestricted forms of the two new generalized Wahba problems. The sixth section summarizes the paper's results and gives its conclusions. The Appendix contains some of the details of the gradient-based analysis presented in the fourth section.

\section{Two Generalized Wahba Problems for a Spinning Spacecraft}

This section reviews the original form of Wahba's problem and the $q$-method solution. It then extends the Wahba problem to develop two new problems that are applicable to a spinning spacecraft whose attitude and rate must be estimated based on a time series of vector attitude observations.

\section{Review of Wahba's Problem}

The original Wahba problem seeks to determine the attitude of a spacecraft at a single instant of time based in body axis measurements of two or more unit direction vectors [5]. The estimation problem can be posed as the constrained optimization problem 


$$
\begin{array}{ll}
\text { find: } & \mathbf{q} \\
\text { to minimize: } & J(\mathbf{q})=\frac{1}{2} \sum_{i=1}^{m} \frac{1}{\sigma_{i}^{2}}\left[\mathbf{b}_{i}-A(\mathbf{q}) \mathbf{r}_{i}\right]^{\mathrm{T}}\left[\mathbf{b}_{i}-A(\mathbf{q}) \mathbf{r}_{i}\right] \\
\text { subject to: } & \mathbf{q}^{\mathrm{T}} \mathbf{q}=1
\end{array}
$$

where $\mathbf{q}$ is the quaternion that parameterizes the attitude. The function $J(\mathbf{q})$ is the least-squares cost function that penalizes errors between the body-axis measured unit direction vectors $\mathbf{b}_{i}$ and the transformations of the corresponding unit reference vectors $\mathbf{r}_{i}$ from inertial coordinates to body coordinates using the direction cosines matrix $A(\mathbf{q})$. The $q$ method transforms this problem into the quadratic maximization problem

$$
\begin{array}{ll}
\text { find: } & \mathbf{q} \\
\text { to minimize: } & p(\mathbf{q})=\mathbf{q}^{\mathrm{T}} K \mathbf{q} \\
\text { subject to: } & \mathbf{q}^{\mathrm{T}} \mathbf{q}=1
\end{array}
$$

where the symmetric $4 \times 4$ matrix $K$ takes the form

$$
K=\sum_{i-1}^{m} K_{i}
$$

with the $4 \times 4$ component matrices $K_{i}$ defined as [3,6]

$$
K_{i}=\frac{1}{\sigma_{i}^{2}}\left[\begin{array}{cc}
\left\{\mathbf{r}_{i} \mathbf{b}_{i}^{\mathrm{T}}+\mathbf{b}_{i} \mathbf{r}_{i}^{\mathrm{T}}-I_{3 \times 3}\left(\mathbf{b}_{i}^{\mathrm{T}} \mathbf{r}_{i}\right)\right\} & \left(\mathbf{b}_{i} \times \mathbf{r}_{i}\right) \\
\left(\mathbf{b}_{i} \times \mathbf{r}_{i}\right)^{\mathrm{T}} & \mathbf{b}_{i}^{\mathrm{T}} \mathbf{r}_{i}
\end{array}\right]
$$

One of the most significant aspects of the Wahba problem is that it can be solved in closed-form by solving an eigenvalue problem. This approach is known as the $q$ method solution [3,6]. If one adjoins the constraint in equation (2c) to the performance function in equation (2b) using the Lagrange multiplier $\lambda$, then the first-order necessary conditions for maximizing $p(\mathbf{q})$ take the form

$$
\begin{aligned}
K \mathbf{q} & =\lambda \mathbf{q} \\
\mathbf{q}^{\mathrm{T}} \mathbf{q} & =1
\end{aligned}
$$

which is an eigenvalue problem. The optimal $\mathbf{q}$ is the unit-normalized eigenvector that corresponds to the maximum eigenvalue of $K, \lambda_{\max }$. This solution globally minimizes the original optimal estimation problem in equations (1a)-(1c).

The availability of robust eigenvalue solvers enables the $q$ method to guarantee that the globally optimal attitude has been determined. This ability to guarantee global optimality is a very strong asset of all Wahba/q-method-based estimation algorithms. One of the other leading attitude estimation algorithms, the extended Kalman filter (EKF), can have divergence problems because it relies on linearizations that only have local validity. In other words, it may diverge because it cannot guarantee that its solution is the global optimum of the associated estimation problem [4].

\section{First Generalized Wahba's Problem: Known Spin Direction, Unknown Spin Rate}

The first extension to Wahba's problem relaxes the requirement that all of the measurements be taken at the same instant of time. It includes the estimation of an unknown constant spin rate about the known major inertia axis of the spin-stabilized spacecraft. This generalized Wahba problem can have a solution even if there is no single instant in time when more than one vector measurement is available. 
This generalized Wahba problem is applicable to a spin-stabilized spacecraft that has a nutation damper. Nutation damping ensures that the spacecraft will spin about its major axis of inertia in steady state. Standard spin-balancing techniques imply that this axis will be known in spacecraft body coordinates.

This problem formulation and all of this paper's subsequent developments assume that attitude sensor alignments have been well calibrated in advance. This assumption ensures that the measured $\mathbf{b}_{i}$ vectors are known in the same body-axis coordinate system that is used to define the known spacecraft moment-of-inertia properties.

If one defines the known spin axis to be $\mathbf{e}_{s}=\left[e_{s x} ; e_{s y} ; e_{s z}\right]$, where $\mathbf{e}_{s}$ is a unit direction vector in spacecraft coordinates, and if one defines the unknown constant spin rate to be the scalar $\omega$, then the first generalized Wahba problem takes the form

$$
\begin{array}{ll}
\text { find: } & \mathbf{q}_{0} \text { and } \omega \\
\text { to maximize: } & \bar{p}_{a}\left(\mathbf{q}_{0}, \omega\right)=\mathbf{q}_{0}^{\mathrm{T}}\left\{\sum_{i=1}^{m} \Phi^{\mathrm{T}}\left[\omega\left(t_{i}-t_{0}\right)\right] K_{i} \Phi\left[\omega\left(t_{i}-t_{0}\right)\right]\right\} \mathbf{q}_{0} \\
\text { subject to: } & \mathbf{q}_{0}^{\mathrm{T}} \mathbf{q}_{0}=1
\end{array}
$$

where $\mathbf{q}_{0}$ is the initial attitude quaternion at epoch time $t_{0}, t_{i}$ is the sample time at which the body-vector measurement $\mathbf{b}_{i}$ was made for $i=1, \ldots, m$, and $\Phi\left[\omega\left(t_{i}-t_{0}\right)\right]$ is the orthonormal $4 \times 4$ matrix that maps $\mathbf{q}_{0}$ to $\mathbf{q}\left(t_{i}\right)=$ $\Phi\left[\omega\left(t_{i}-t_{0}\right)\right] \mathbf{q}_{0}$. Given that the spin vector is known to be constant and equal to $\omega \mathbf{e}_{s}$, the quaternion dynamics model in reference [6] implies that this matrix is

$\Phi\left[\omega\left(t_{i}-t_{0}\right)\right]=I_{4 \times 4} \cos \left[\frac{\omega\left(t_{i}-t_{0}\right)}{2}\right]+\left[\begin{array}{cccc}0 & e_{s z} & -e_{s y} & e_{s x} \\ -e_{s z} & 0 & e_{s x} & e_{s y} \\ e_{s y} & -e_{s x} & 0 & e_{s z} \\ -e_{s x} & -e_{s y} & -e_{s z} & 0\end{array}\right] \sin \left[\frac{\omega\left(t_{i}-t_{0}\right)}{2}\right]$

This generalized Wahba problem differs markedly from previous time-varying Wahba problems that have been proposed, as typified by the problem defined in references [9] and [10]. Such problems presume the availability of three-axis rate-gyro data. Although they include a quaternion transition matrix $\Phi\left(t_{i}, t_{0}\right)$ in an estimation performance metric, as in equation $(6 \mathrm{~b})$, their $\Phi\left(t_{i}, t_{0}\right)$ matrix does not depend on any unknown spin rate. It is assumed to be known from the gyro data and from integration of the associated transition matrix differential equation.

The estimation performance metric in equation (6b) can be re-written in the form $\bar{p}_{a}\left(\mathbf{q}_{0}, \omega\right)=\mathbf{q}_{0}^{\mathrm{T}} K_{a}(\omega) \mathbf{q}_{0}$ where

$$
K_{a}(\omega)=\sum_{i=1}^{m} \Phi^{\mathrm{T}}\left[\omega\left(t_{i}-t_{0}\right)\right] K_{i} \Phi\left[\omega\left(t_{i}-t_{0}\right)\right]
$$

Thus, the new problem form retains the original $q$-method form. The only difference is that its $K$ matrix now depends on an unknown parameter.

\section{Second Generalized Wahba's Problem: Known Inertia Matrix and Torque-Free Motion}

The second generalized Wahba problem for a spinning spacecraft relaxes the requirements that the spin axis is known a priori and that the spin rate is constant. It replaces these assumptions with assumptions that the spacecraft is rigid, that its 
$3 \times 3$ body-axis moment-of-inertia matrix $I_{s c}$ is known, and that the spacecraft undergoes torque-free motion. Thus, the spacecraft can nutate while it spins. The corresponding generalized form of the Wahba problem is

$$
\begin{array}{ll}
\text { find: } & \mathbf{q}_{0} \text { and } \boldsymbol{\omega}_{0} \\
\text { to maximize: } & \bar{p}_{b}\left(\mathbf{q}_{0}, \boldsymbol{\omega}_{0}\right)=\mathbf{q}_{0}^{\mathrm{T}}\left[\sum_{i=1}^{m} \Phi^{\mathrm{T}}\left(t_{i}, t_{0} ; \boldsymbol{\omega}_{0}\right) K_{i} \Phi\left(t_{i}, t_{0} ; \boldsymbol{\omega}_{0}\right)\right] \mathbf{q}_{0} \\
\text { subject to: } & \mathbf{q}_{0}^{\mathrm{T}} \mathbf{q}_{0}=1
\end{array}
$$

where the unknown $3 \times 1$ vector $\boldsymbol{\omega}_{0}$ is the initial angular velocity of the spacecraft at time $t_{0}$ expressed in body coordinates, and $\Phi\left(t_{i}, t_{0} ; \boldsymbol{\omega}_{0}\right)$ is the quaternion transition matrix that is used to determine $\mathbf{q}\left(t_{i}\right)=\Phi\left(t_{i}, t_{0} ; \boldsymbol{\omega}_{0}\right) \mathbf{q}_{0}$ for a rigid body undergoing torque-free motion. This matrix can be determined by integrating Euler's equation to determine $\boldsymbol{\omega}(t)=\left[\omega_{x}(t) ; \omega_{y}(t) ; \omega_{z}(t)\right]$ as a function of $\boldsymbol{\omega}_{0}$ while simultaneously integrating the quaternion transition matrix differential equation from the initial condition $\Phi\left(t_{0}, t_{0} ; \boldsymbol{\omega}_{0}\right)=I_{4 \times 4}$. This system of equations takes the wellknown form [6]

$$
\begin{aligned}
\frac{\mathrm{d} \boldsymbol{\omega}}{\mathrm{d} t} & =-I_{s c}^{-1}\left[\boldsymbol{\omega} \times\left(I_{s c} \boldsymbol{\omega}\right)\right] \\
\frac{\mathrm{d} \Phi\left(t, t_{0} ; \boldsymbol{\omega}_{0}\right)}{\mathrm{d} t} & =\frac{1}{2}\left[\begin{array}{cccc}
0 & \omega_{z}(t) & -\omega_{y}(t) & \omega_{x}(t) \\
-\omega_{z}(t) & 0 & \omega_{x}(t) & \omega_{y}(t) \\
\omega_{y}(t) & -\omega_{x}(t) & 0 & \omega_{z}(t) \\
-\omega_{x}(t) & -\omega_{y}(t) & -\omega_{z}(t) & 0
\end{array}\right] \Phi\left(t, t_{0} ; \boldsymbol{\omega}_{0}\right)
\end{aligned}
$$

Note that $I_{s c}$ can be assumed to be diagonal without loss of generality.

This system of equations can be integrated numerically or analytically. The result in equation (7) is the solution of this system of equations for the special case where $\mathbf{e}_{s}$ is a principal axis of $I_{s c}$ and $\boldsymbol{\omega}_{0}$ is aligned with this axis. A more complicated closed-form solution for $\Phi\left(t, t_{0} ; \boldsymbol{\omega}_{0}\right)$ can be determined for the case of axial symmetry. It exploits the notion of a body-fixed cone rolling on a space cone and can be written in terms of ordinary trigonometric functions ([6], p. 530). The solution for the general case involves the use of Serret-Andoyer variables [13] and is very complex. It is not clear that an analytic form of this latter solution would serve better within an optimization algorithm than would a solution based on numerical integration of equations (10a) and (10b).

Similar to the first generalized Wahba problem, the performance metric in equation (9b) can be recast into the form $\bar{p}_{b}\left(\mathbf{q}_{0}, \boldsymbol{\omega}_{0}\right)=\mathbf{q}_{0}^{\mathrm{T}} K_{b}\left(\boldsymbol{\omega}_{0}\right) \mathbf{q}_{0}$ where

$$
K_{b}\left(\boldsymbol{\omega}_{0}\right)=\sum_{i=1}^{m} \Phi^{\mathrm{T}}\left(t_{i}, t_{0} ; \boldsymbol{\omega}_{0}\right) K_{i} \Phi\left(t_{i}, t_{0} ; \boldsymbol{\omega}_{0}\right)
$$

Again, this is the problem form that is addressed by the original $q$ method, except that the $K$ matrix is a function of the initial angular velocity vector $\boldsymbol{\omega}_{0}$.

It may be beneficial to reformulate the 2 nd generalized Wahba problem by replacing the unknown initial angular velocity $\boldsymbol{\omega}_{0}$ with the unknown initial angular momentum. One could use the initial angular momentum in spacecraft coordinates, $\mathbf{h}_{s c 0}=I_{s c} \boldsymbol{\omega}_{0}$, or the constant angular momentum in inertial coordinates: $\mathbf{h}_{i n}=A^{\mathrm{T}}\left[\mathbf{q}_{0}\right] I_{s c} \boldsymbol{\omega}_{0}$. There is a one-to-one relationship between the vector of unknowns $\left[\mathbf{q}_{0} ; \boldsymbol{\omega}_{0}\right]$ in the original formulation of this generalized problem and the two 
proposed new vectors of unknowns $\left[\mathbf{q}_{0} ; \mathbf{h}_{s c 0}\right]$ and $\left[\mathbf{q}_{0} ; \mathbf{h}_{\text {in }}\right]$. It is straightforward to transform the problem between these three different possible formulations. The resulting transformations would yield Wahba-type performance metrics of the form $\bar{p}_{c}\left(\mathbf{q}_{0}, \mathbf{h}_{s c 0}\right)=\mathbf{q}_{0}^{\mathrm{T}} K_{c}\left(\mathbf{h}_{s c 0}\right) \mathbf{q}_{0}$ or $\bar{p}_{d}\left(\mathbf{q}_{0}, \mathbf{h}_{i n}\right)=\mathbf{q}_{0}^{\mathrm{T}} K_{d}\left(\mathbf{q}_{0}, \mathbf{h}_{i n}\right) \mathbf{q}_{0}$. The calculation of the symmetric matrix $K_{c}\left(\mathbf{h}_{s c 0}\right)$ or $K_{d}\left(\mathbf{q}_{0}, \mathbf{h}_{i n}\right)$ would involve integration of the corresponding versions of equations (10a) and (10b) followed by a summation like that in equation (11). The $\bar{p}_{d}\left(\mathbf{q}_{0}, \mathbf{h}_{i n}\right)$ performance metric is probably less useful. The added dependence of $K_{d}\left(\mathbf{q}_{0}, \mathbf{h}_{i n}\right)$ on $\mathbf{q}_{0}$ would complicate any effort to maximize $\bar{p}_{d}\left(\mathbf{q}_{0}, \mathbf{h}_{i n}\right)$.

\section{A Restricted Version of the First Generalized Wahba Problem and its Exact Solution}

A restricted version of the first Wahba problem in equations $(6 a)-(6 c)$ can be solved exactly. This problem limits the number of vector measurements to the minimum that is needed in order to make the solution unique: $m=2$ measurements at two distinct measurement times $t_{1} \neq t_{2}$ with reference unit direction vectors $\mathbf{r}_{1}$ and $\mathbf{r}_{2}$ that are linearly independent. This restricted problem is observable in the general case because each measurement contains two independent pieces of scalar information, making for a total of four equivalent scalar measurements. The problem unknowns also constitute the equivalent of four scalars, the three free parameters in the quaternion $\mathbf{q}_{0}$ after its normalization constraint has been considered and the spin rate $\omega$. Therefore, the four problem unknowns should be determinable from the four pieces of attitude information in the two vector measurements.

The solution of the restricted problem amounts to equation solving. It produces an equivalent to the equation (1b) cost that is exactly zero. This solution starts by recognizing that each matrix $K_{i}$, as defined in equation (4), has two equal maximum eigenvalues with two orthogonal eigenvectors. Using the results of reference [14], this subspace of eigenvectors is spanned by the two quaternions

$$
\begin{aligned}
\mathbf{q}_{\alpha i} & =\left\{\begin{array}{cc}
\frac{1}{\sqrt{2\left(1+\mathbf{b}_{i}^{\mathrm{T}} \mathbf{r}_{i}\right)}}\left[\begin{array}{c}
\mathbf{b}_{i} \times \mathbf{r}_{i} \\
\left(1+\mathbf{b}_{i}^{\mathrm{T}} \mathbf{r}_{i}\right)
\end{array}\right] & \text { if }-1<\mathbf{b}_{i}^{\mathrm{T}} \mathbf{r}_{i} \\
{\left[\begin{array}{c}
\mathbf{c}_{i} \\
\mathbf{0}
\end{array}\right]} & \text { if }-1=\mathbf{b}_{i}^{\mathrm{T}} \mathbf{r}_{i}
\end{array}\right. \\
\mathbf{q}_{\beta i} & =\left[\begin{array}{c}
\mathbf{b}_{i} \\
0
\end{array}\right] \otimes \mathbf{q}_{\alpha i}
\end{aligned}
$$

where $\mathbf{c}_{i}$ in the lower condition of equation (12a) is any vector that is perpendicular to $\mathbf{b}_{i}$ and where standard quaternion multiplication [6] is used in equation (12b) with the order convention such that $A\left(\mathbf{q}_{a} \otimes \mathbf{q}_{b}\right)=A\left(\mathbf{q}_{a}\right) A\left(\mathbf{q}_{b}\right)$. The general maximizing eigenvector is the quaternion

$$
\mathbf{q}\left(t_{i}\right)=\mathbf{q}_{\alpha i} \cos \left(\theta_{i}\right)+\mathbf{q}_{\beta i} \sin \left(\theta_{i}\right)
$$

The angle $\theta_{i}$ is a free parameter that will be determined by the solution procedure in a way that causes $\mathbf{q}\left(t_{i}\right)$ to equal the optimal estimate of the attitude quaternion at time $t_{i}$.

The quaternion formula in equation (13) can be applied at the measurement times $t_{1}$ and $t_{2}$, and the results can be used in a quaternion multiplication in order to determine the quaternion of rotation between these two times as 


$$
\begin{aligned}
\mathbf{q}_{\text {rot } 21}= & \mathbf{q}\left(t_{2}\right) \otimes \mathbf{q}^{-1}\left(t_{1}\right) \\
= & {\left[\mathbf{q}_{\alpha 2} \cos \left(\theta_{2}\right)+\mathbf{q}_{\beta 2} \sin \left(\theta_{2}\right)\right] \otimes\left[\mathbf{q}_{\alpha 1}^{-1} \cos \left(\theta_{1}\right)+\mathbf{q}_{\beta 1}^{-1} \sin \left(\theta_{1}\right)\right] } \\
= & \cos \left(\theta_{2}+\theta_{1}\right) \frac{1}{2}\left[\mathbf{q}_{\alpha 2} \otimes \mathbf{q}_{\alpha 1}^{-1}-\mathbf{q}_{\beta 2} \otimes \mathbf{q}_{\beta 1}^{-1}\right] \\
& +\sin \left(\theta_{2}+\theta_{1}\right) \frac{1}{2}\left[\mathbf{q}_{\alpha 2} \otimes \mathbf{q}_{\beta 1}^{-1}+\mathbf{q}_{\beta 2} \otimes \mathbf{q}_{\alpha 1}^{-1}\right] \\
& +\cos \left(\theta_{2}-\theta_{1}\right) \frac{1}{2}\left[\mathbf{q}_{\alpha 2} \otimes \mathbf{q}_{\alpha 1}^{-1}+\mathbf{q}_{\beta 2} \otimes \mathbf{q}_{\beta 1}^{-1}\right] \\
& +\sin \left(\theta_{2}-\theta_{1}\right) \frac{1}{2}\left[-\mathbf{q}_{\alpha 2} \otimes \mathbf{q}_{\beta 1}^{-1}+\mathbf{q}_{\beta 2} \otimes \mathbf{q}_{\alpha 1}^{-1}\right]
\end{aligned}
$$

The quaternion notation $\mathbf{q}^{-1}$ indicates the quaternion formed by negating the first three elements of $\mathbf{q}$ while keeping the fourth element unchanged. It constitutes the inverse rotation so that $\mathbf{q} \otimes \mathbf{q}^{-1}=\mathbf{q}^{-1} \otimes \mathbf{q}=[0 ; 0 ; 0 ; 1]$, the identity quaternion.

The solution to the restricted first problem makes use of the fact that the rotation quaternion in equation (14) must equal

$$
\mathbf{q}_{\text {rot 21 theory }}=\left[\begin{array}{c}
\mathbf{e}_{s} \sin \left(\frac{\omega\left(t_{2}-t_{1}\right)}{2}\right) \\
\cos \left(\frac{\omega\left(t_{2}-t_{1}\right)}{2}\right)
\end{array}\right]
$$

In particular, the first three elements of $\mathbf{q}_{\text {rot2 }}$ from equation (14) must be parallel to the spin direction vector $\mathbf{e}_{s}$. One can choose two unit direction vectors $\mathbf{e}_{\perp a}$ and $\mathbf{e}_{\perp b}$ such that $\left[\mathbf{e}_{\perp a}, \mathbf{e}_{\perp b}, \mathbf{e}_{s}\right]$ forms an orthonormal triad. One uses these two vectors to develop a pair of equations for the two unknown angles $\phi=\theta_{2}+\theta_{1}$ and $\gamma=\theta_{2}-\theta_{1}$. These equations take the form

$$
0=\left[\begin{array}{ll}
\mathbf{e}_{\perp a}^{\mathrm{T}} & 0 \\
\mathbf{e}_{\perp b}^{\mathrm{T}} & 0
\end{array}\right] q_{\text {rot } 21}=F\left[\begin{array}{c}
\cos \phi \\
\sin \phi
\end{array}\right]+G\left[\begin{array}{c}
\cos \gamma \\
\sin \gamma
\end{array}\right]
$$

where the $2 \times 2$ matrices $F$ and $G$ are

$$
\begin{aligned}
& F=\frac{1}{2}\left[\begin{array}{ll}
\mathbf{e}_{\perp a}^{\mathrm{T}} & 0 \\
\mathbf{e}_{\perp b}^{\mathrm{T}} & 0
\end{array}\right]\left[\left(\mathbf{q}_{\alpha 2} \otimes \mathbf{q}_{\alpha 1}^{-1}-\mathbf{q}_{\beta 2} \otimes \mathbf{q}_{\beta 1}^{-1}\right),\left(\mathbf{q}_{\alpha 2} \otimes \mathbf{q}_{\beta 1}^{-1}+\mathbf{q}_{\beta 2} \otimes \mathbf{q}_{\alpha 1}^{-1}\right)\right] \\
& G=\frac{1}{2}\left[\begin{array}{ll}
\mathbf{e}_{\perp a}^{\mathrm{T}} & 0 \\
\mathbf{e}_{\perp b}^{\mathrm{T}} & 0
\end{array}\right]\left[\left(\mathbf{q}_{\alpha 2} \otimes \mathbf{q}_{\alpha 1}^{-1}+\mathbf{q}_{\beta 2} \otimes \mathbf{q}_{\beta 1}^{-1}\right),\left(-\mathbf{q}_{\alpha 2} \otimes \mathbf{q}_{\beta 1}^{-1}+\mathbf{q}_{\beta 2} \otimes \mathbf{q}_{\alpha 1}^{-1}\right)\right]
\end{aligned}
$$

Equation (16) is used to solve for the $2 \times 1$ vector [ $\cos \phi$; $\sin \phi]$ in terms of the vector $[\cos \gamma ; \sin \gamma]$, and the result is substituted into the equation $\cos ^{2} \phi+$ $\sin ^{2} \phi=1$ to yield the equation

$$
1=[\cos \gamma \sin \gamma] G^{\mathrm{T}}\left(F^{-1}\right)^{\mathrm{T}} F^{-1} G\left[\begin{array}{c}
\cos \gamma \\
\sin \gamma
\end{array}\right]=[\cos \gamma \sin \gamma] L\left[\begin{array}{l}
\cos \gamma \\
\sin \gamma
\end{array}\right]
$$

where $L=G^{\mathrm{T}}\left(F^{-1}\right)^{\mathrm{T}} F^{-1} G$ is a symmetric $2 \times 2$ matrix. In the general case, $F$ is conjectured to have an inverse, as demonstrated by several randomly developed numerical examples, all of which yielded a nonsingular $F$ matrix. If $F$ is not invertible, then it should be possible to develop an alternate equation in terms of $\cos \phi$ and $\sin \phi$ and 
the inverse of $G$, one that solves equation (16) for the $2 \times 1$ vector $[\cos \gamma$; $\sin \gamma]$ in terms of the vector $[\cos \phi ; \sin \phi]$ and that substitutes the result into the equation $\cos ^{2} \gamma+\sin ^{2} \gamma=1$. Alternatively, it may be possible to choose the vectors $\mathbf{e}_{\perp a}$ and $\mathbf{e}_{\perp b}$ in a way that ensures the nonsingularity of $F$. The conditions that ensure nonsingularity of $F$ or $G$ constitute a subject that warrants further investigation.

Trigonometric identities can be used to re-cast equation (18) in the form

$$
1-\left(\frac{L_{11}+L_{22}}{2}\right)=u \sin (2 \gamma+\psi)
$$

where

$$
\begin{aligned}
& u=\sqrt{L_{12}^{2}+\left(\frac{L_{11}-L_{22}}{2}\right)^{2}} \\
& \psi=\operatorname{atan} 2\left[\left(\frac{L_{11}-L_{22}}{2}\right), L_{12}\right]
\end{aligned}
$$

and where atan2[,] is the usual two-argument arctangent function. Equation (19) can be solved in closed form. It has two solutions in the form of

$$
\begin{aligned}
& \gamma=\frac{1}{2}\left\{\operatorname{asin}\left[\frac{1-\frac{1}{2}\left(L_{11}+L_{22}\right)}{u}\right]-\psi\right\}+n \pi \\
& \gamma=\frac{1}{2}\left\{\pi-\operatorname{asin}\left[\frac{1-\frac{1}{2}\left(L_{11}+L_{22}\right)}{u}\right]-\psi\right\}+n \pi
\end{aligned}
$$

The $n \pi$ ambiguities for integer-valued $n$ only affect the common-mode signs of the elements of the quaternions $\mathbf{q}\left(t_{i}\right)$ in equation (13). Such sign changes do not change the represented attitudes; therefore, nonzero values of $n$ can be ignored.

The ability to distinguish which of these two solutions is correct is not provided by the data at the two sample times that are used in this restricted problem. Numerical truth-model simulations show that a simple test involving a third vector observation at a third sample time suffices to resolve this ambiguity.

Given the correct $\gamma$ from equation (21a) or (21b), a back substitution procedure is needed in order to determine the other solution quantities of interest. This procedure starts by substituting $\gamma$ into equation (16) and solving for $\phi$. The quantities $\theta_{1}=$ $0.5(\phi-\gamma)$ and $\theta_{2}=0.5(\phi+\gamma)$ are computed next, and these quantities are substituted into equation (13) in order to compute $\mathbf{q}\left(t_{1}\right)$ and $\mathbf{q}\left(t_{2}\right)$. These quaternions are substituted into the first line of equation (14) in order to compute the rotation quaternion $\mathbf{q}_{r o t 21}$. This quaternion is equated with the theoretical formula in equation (15) in order to compute the spin rate $\omega$. Finally, this spin rate is substituted into equation (7) in order to compute the quaternion transition matrix $\Phi\left[\omega\left(t_{1}-t_{0}\right)\right]$, and this matrix is used to compute the initial quaternion: $\mathbf{q}_{0}=\Phi^{\mathrm{T}}\left[\omega\left(t_{1}-t_{0}\right)\right] \mathbf{q}\left(t_{1}\right)$.

This solution procedure has been encoded and tested on simulated truth-model problems. Except for the ambiguity noted between the two $\gamma$ values in equations (21a) and (21b), the numerical calculations bear out the correctness of this solution.

This solution to the restricted form of the first generalized Wahba problem could be useful for initializing an EKF. Given its global optimality, its solution would be likely to be very near the true attitude and spin rate if the measurements were reasonably accurate. This good solution could provide an EKF with enough initialization accuracy to avoid the possibility of divergence due to linearization errors. 
This solution may provide insight that could lead to the development of a closedform solution to the unrestricted version of the first generalized Wahba problem. Alternatively, it could provide the starting guess for an iterative numerical solution of the unrestricted problem.

\section{A Restricted Form of the Second Generalized Wahba Problem}

\section{The Minimum Observable Form of the Second Problem}

The restricted version of the second generalized Wahba problem uses the minimum number of measurements required for observability. Consider the number of free scalar unknowns in the second generalized problem: there are three free parameters in the initial quaternion $\mathbf{q}_{0}$ after the normalization constraint has been enforced, and there are three independent elements of the initial angular velocity vector $\boldsymbol{\omega}_{0}$, which yields a total of six scalar unknowns. Each vector attitude measurement pair $\left(\mathbf{r}_{i}, \mathbf{b}_{i}\right)$ contains two pieces of scalar information. Therefore, at least $m=3$ pairs are needed in order to make the second generalized problem observable. The next two subsections demonstrate that $m=3$ measurements is a sufficient number for observability in a representative set of example cases. The restricted version of the second problem is defined to be the problem that uses this many measurements.

This restricted second generalized Wahba problem may have important practical uses. It may be possible to develop an analytic or semi-analytic global solution to this restricted problem as was done for the first restricted problem. Such a solution could shed light on how best to solve the unrestricted form of the problem. Alternatively, it could be used to initialize one of the EKFs in reference [2], thereby precluding the possibility that the EKF would diverge.

It is possible that initialization of an EKF will be the most important use of either of the generalized Wahba problems. If so, then the restricted forms of the two problems would probably be the most important forms because the corresponding EKF solution might be as good or better than the solution to the unrestricted problem when considered over any significant time duration. In this case, a quest for solutions to the unrestricted forms of the two generalized problems might be more of a pure mathematical challenge than an important contribution to aerospace engineering.

\section{A Second-Order Approximation of the Second Wahba Problem}

A gradient-based approximation of the restricted form of the second generalized Wahba problem can be used to demonstrate its local observability. Suppose that $\left(\mathbf{q}_{\text {oopt }}, \boldsymbol{\omega}_{\text {oopt }}\right)$ is a candidate point for the maximization of the performance metric $\bar{p}_{b}\left(\mathbf{q}_{0}, \boldsymbol{\omega}_{0}\right)$ of equation (9b). Then the optimal estimation problem in equations $(9 a)-(9 c)$ can be approximated to second order near this point by the problem

find: $\quad \Delta \mathbf{q}_{0}$ and $\Delta \boldsymbol{\omega}_{0}$

to maximize: $\Delta \bar{p}_{b}\left(\Delta \mathbf{q}_{0}, \Delta \boldsymbol{\omega}_{0}\right)=\left[\mathbf{g}_{q}^{\mathrm{T}}, \mathbf{g}_{\omega}^{\mathrm{T}}\right]\left[\begin{array}{c}\Delta \mathbf{q}_{0} \\ \Delta \boldsymbol{\omega}_{0}\end{array}\right]$

$$
+\frac{1}{2}\left[\Delta \mathbf{q}_{0}^{\mathrm{T}}, \Delta \boldsymbol{\omega}_{0}^{\mathrm{T}}\right]\left[\begin{array}{cc}
H_{q q}-2 \lambda_{\text {opt }} I_{4 \times 4} & H_{q \omega} \\
H_{q \omega}^{\mathrm{T}} & H_{\omega \omega}
\end{array}\right]\left[\begin{array}{c}
\Delta \mathbf{q}_{0} \\
\Delta \boldsymbol{\omega}_{0}
\end{array}\right]
$$

subject to: $\quad \mathbf{q}_{\text {oopt }}^{\mathrm{T}} \Delta \mathbf{q}_{0}=0$ 
where $\Delta \mathbf{q}_{0}$ and $\Delta \boldsymbol{\omega}_{0}$ are perturbations to $\mathbf{q}_{0 o p t}$ and $\boldsymbol{\omega}_{0 o p t}$ and where $\Delta \bar{p}_{b}\left(\Delta \mathbf{q}_{0}, \Delta \boldsymbol{\omega}_{0}\right)$ is the second order approximation of the corresponding perturbation to $\bar{p}_{b}\left(\mathbf{q}_{\text {oopt }}, \boldsymbol{\omega}_{\text {oopt }}\right)$. The variable $\lambda_{\text {opt }}=\mathbf{q}_{\text {oopt }}^{\mathrm{T}} K_{b}\left(\boldsymbol{\omega}_{0 \text { opt }}\right) \mathbf{q}_{\text {oopt }}$ is the Lagrange multiplier for the constraint in equation (9c) and equals the largest eigenvalue of $K_{b}\left(\boldsymbol{\omega}_{0 o p t}\right)$ because $\mathbf{q}_{0 o p t}$ must equal the corresponding eigenvector. The vectors $\mathbf{g}_{q}$ and $\mathbf{g}_{\omega}$ are the gradients of $\bar{p}_{b}\left(\mathbf{q}_{0}, \boldsymbol{\omega}_{0}\right)$ with respect to $\mathbf{q}_{0}$ and $\boldsymbol{\omega}_{0}$, and the matrices $H_{q q}, H_{q \omega}$, and $H_{\omega \omega}$ are the Hessian second partial derivatives of $\bar{p}_{b}\left(q_{0}, \boldsymbol{\omega}_{0}\right)$ with respect to $\mathbf{q}_{0}$ and $\boldsymbol{\omega}_{0}$, all evaluated at $\mathbf{q}_{\text {oopt }}$ and $\boldsymbol{\omega}_{0 \text { opt }}$. The Appendix presents formulas for the computation of $\mathbf{g}_{q}, \mathbf{g}_{\omega}, H_{q q}$, $H_{q \omega}$, and $H_{\omega \omega}$.

\section{Local Observability of the Restricted Second Problem}

A system is said to be observable if and only if there exists a unique solution to the corresponding well-determined or over-determined system of equations that incorporates the system's measurement model and dynamic model. Local observability of the second generalized Wahba problem is the equivalent of demonstrating that the problem in equations (22a) $-\left(22 \mathrm{c}\right.$ ) has the unique global maximum $\Delta \mathbf{q}_{0}=0$ and $\Delta \boldsymbol{\omega}_{0}=0$. Two conditions must be met for uniqueness to hold in this case. They are the first-order necessary condition and the second-order sufficient condition for $\left(\mathbf{q}_{\text {oopt }}, \boldsymbol{\omega}_{0 \text { opt }}\right)$ to be a local maximum of the original problem in equations $(9 \mathrm{a})-(9 \mathrm{c})$.

The first-order necessary condition requires that $\mathbf{g}_{q}-\beta \mathbf{q}_{\text {oopt }}=0$ and that $\mathbf{g}_{\omega}=0$. The scalar $\beta$ is the Lagrange multiplier for the linearized constraint in equation (22c). A review of the formula for $\mathbf{g}_{q}$ in equation (A1a) of the Appendix shows that $\beta=2 \lambda_{\text {opt }}$ will cause the first of these two equations to be satisfied if $\mathbf{q}_{\text {oopt }}$ is an eigenvector of $K_{b}\left(\boldsymbol{\omega}_{0 \text { opt }}\right)$ with eigenvalue equal to $\lambda_{\text {opt }}$. This is a familiar result from the original $q$-method solution of the Wahba problem. The equation $\mathbf{g}_{\omega}=0$ is a new condition imposed by the present paper's generalization of Wahba's problem. For purposes of the local observability analysis, it can be assumed that the chosen $\left(\mathbf{q}_{\text {oopt }}, \boldsymbol{\omega}_{0 \text { opt }}\right)$ point satisfies these two first-order necessary conditions.

The second-order sufficient condition for a local maximum at the point $\left(\mathbf{q}_{\text {oopt }}, \boldsymbol{\omega}_{0 \text { opt }}\right)$ is that the projected Hessian of the performance metric $\Delta \bar{p}_{b}\left(\Delta \mathbf{q}_{0}, \Delta \boldsymbol{\omega}_{0}\right)$ be negative definite. The Hessian matrix is the $7 \times 7$ block matrix in the last term on the right-hand side of equation (22b). The second-order sufficient condition considers the projection of this Hessian into the six-dimensional subspace of $\left[\Delta \mathbf{q}_{0}, \Delta \boldsymbol{\omega}_{0}\right]$ space that is perpendicular to the constraint gradient vector $\left[\mathbf{q}_{\text {oopt }} ; 0\right]$ Negative definiteness of the projected Hessian implies that every nonzero vector $\left[\Delta \mathbf{q}_{0}, \Delta \boldsymbol{\omega}_{0}\right]$ produces a negative value when substituted into the last term on the right-hand side of equation (22b) if the vector's $\Delta \mathbf{q}_{0}$ component obeys $\mathbf{q}_{\text {Oopt }}^{\mathrm{T}} \Delta \mathbf{q}_{0}=0$.

A suitable projection of the Hessian can be calculated by first determining a $4 \times 3$ projection matrix $Q_{\perp}$ such that the $4 \times 4$ matrix $\left[Q_{\perp}, \mathbf{q}_{\text {oopt }}\right]$ is orthonormal. One possible choice of $Q_{\perp}$ is

$$
Q_{\perp}=\left[\begin{array}{ccc}
\left(\mathbf{q}_{\text {oopt }}\right)_{4} & \left(\mathbf{q}_{\text {oopt }}\right)_{3} & -\left(\mathbf{q}_{\text {oopt }}\right)_{2} \\
-\left(\mathbf{q}_{\text {oopt }}\right)_{3} & \left(\mathbf{q}_{\text {oopt }}\right)_{4} & \left(\mathbf{q}_{\text {oopt }}\right)_{1} \\
\left(\mathbf{q}_{\text {oopt }}\right)_{2} & -\left(\mathbf{q}_{\text {oopt }}\right)_{1} & \left(\mathbf{q}_{\text {oopt }}\right)_{4} \\
-\left(\mathbf{q}_{\text {oopt }}\right)_{1} & -\left(\mathbf{q}_{\text {oopt }}\right)_{2} & -\left(\mathbf{q}_{\text {oopt }}\right)_{3}
\end{array}\right]
$$

as suggested by quaternion multiplication theory [6]. Given this projection, the required $6 \times 6$ projected Hessian matrix is 


$$
H_{p r o j}=\left[\begin{array}{cc}
Q_{\perp}^{\mathrm{T}}\left(H_{q q}-2 \lambda_{\text {opt }} I_{4 \times 4}\right) Q_{\perp} & Q_{\perp}^{\mathrm{T}} H_{q \omega} \\
H_{q \omega}^{\mathrm{T}} Q_{\perp} & H_{\omega \omega}
\end{array}\right]
$$

The second-order sufficient condition can be evaluated by computing the six realvalued eigenvalues of the symmetric $H_{\text {proj }}$ matrix. It is negative definite and the system is locally observable if and only if all six eigenvalues are negative.

The second-order problem approximation and observability calculations of equations (22a)-(24) have been carried out numerically for several example cases. These computations have used "truth" values of $\mathbf{q}_{0}$ and $\boldsymbol{\omega}_{0}$ in order to simulate the $\mathbf{b}_{i}$ vector measurements. The measurements have been simulated with zero measurement noise. The same truth values of $\mathbf{q}_{0}$ and $\boldsymbol{\omega}_{0}$ have then been used as $\mathbf{q}_{0 \text { opt }}$ and $\boldsymbol{\omega}_{0 \text { opt }}$ in equations (22a)-(24). This guarantees that the first-order necessary conditions for a local maximum are satisfied. The question of local observability has been answered by computing the six eigenvalues of $H_{\text {proj }}$ and checking that they are all negative. A variety of truth values of $\mathbf{q}_{0}$ and $\boldsymbol{\omega}_{0}$ have been generated for these tests with the aid of a random number generator.

These calculations have confirmed the observability of the restricted form of the second generalized Wahba problem for all of the numerical cases that have been considered. Recall that this restricted problem is the form that incorporates the minimum required number of vector measurements, $m=3$. This numerical evidence demonstrates observability in the general case that has three different measurement times $t_{1}, t_{2}$, and $t_{3}$ and three linearly independent inertial reference vectors $\mathbf{r}_{1}, \mathbf{r}_{2}$, and $\mathbf{r}_{3}$. The numerical evidence also shows that the problem can retain observability even when two of the reference vectors are the same while the third is linearly independent, i.e., when $\mathbf{r}_{1}=\mathbf{r}_{2}, \mathbf{r}_{1}=\mathbf{r}_{3}$, or $\mathbf{r}_{2}=\mathbf{r}_{3}$. The restricted problem loses observability, however, if all three inertial reference vectors lie in the same direction or if two or more of the measurement times are the same.

There might exist isolated additional cases in which the restricted problem is not observable, perhaps cases where the sample times induce an aliasing-like effect with respect to the spacecraft's nutation or spin frequency. No such perverse cases have yet been encountered.

\section{Possible Analytic Solution Strategies for the Restricted Second Problem} in the Case of Axial Symmetry

It would be useful to have an analytic global solution to the restricted version of the second generalized Wahba problem. Such a solution would be for the second problem what the analytic solution of the third section is for the first problem. No such solution has yet been derived. This subsection suggests strategies that might be employed to derive one.

The first step in deriving an analytic solution is to develop an analytic expression for the $\Phi\left(t, t_{0} ; \boldsymbol{\omega}_{0}\right)$ quaternion state transition matrix. It is probably possible to derive an analytic expression for $\Phi\left(t, t_{0} ; \boldsymbol{\omega}_{0}\right)$ for a general moment-of-inertia matrix $I_{S C}[13]$, but the expression is likely to be too complicated to help in deriving an analytic solution to the restricted second generalized Wahba problem. In the case of axial symmetry, however, the expression for $\Phi\left(t, t_{0} ; \boldsymbol{\omega}_{0}\right)$ is relatively simple. It can be derived in terms of two rotations that model the rolling of the "body cone" on the "space cone," as discussed in reference [6] on pp. 491-492. This expression takes the form (reference [6], p. 530) 


$$
\Phi\left(t, t_{0} ; \boldsymbol{\omega}_{0}\right)=\left[\begin{array}{cccc}
q_{\Phi 4}\left(t-t_{0}, \boldsymbol{\omega}_{0}\right) & q_{\Phi 3}\left(t-t_{0}, \boldsymbol{\omega}_{0}\right) & -q_{\Phi 2}\left(t-t_{0}, \boldsymbol{\omega}_{0}\right) & q_{\Phi 1}\left(t-t_{0}, \boldsymbol{\omega}_{0}\right) \\
-q_{\Phi 3}\left(t-t_{0}, \boldsymbol{\omega}_{0}\right) & q_{\Phi 4}\left(t-t_{0}, \boldsymbol{\omega}_{0}\right) & q_{\Phi 1}\left(t-t_{0}, \boldsymbol{\omega}_{0}\right) & q_{\Phi 2}\left(t-t_{0}, \boldsymbol{\omega}_{0}\right) \\
q_{\Phi 2}\left(t-t_{0}, \boldsymbol{\omega}_{0}\right) & -q_{\Phi 1}\left(t-t_{0}, \boldsymbol{\omega}_{0}\right) & q_{\Phi 4}\left(t-t_{0}, \boldsymbol{\omega}_{0}\right) & q_{\Phi 3}\left(t-t_{0}, \boldsymbol{\omega}_{0}\right) \\
-q_{\Phi 1}\left(t-t_{0}, \boldsymbol{\omega}_{0}\right) & -q_{\Phi 2}\left(t-t_{0}, \boldsymbol{\omega}_{0}\right) & -q_{\Phi 3}\left(t-t_{0}, \boldsymbol{\omega}_{0}\right) & q_{\Phi 4}\left(t-t_{0}, \boldsymbol{\omega}_{0}\right)
\end{array}\right]
$$

where

$$
\left[\begin{array}{c}
q_{\Phi 1}\left(t-t_{0}, \boldsymbol{\omega}_{0}\right) \\
q_{\Phi 2}\left(t-t_{0}, \boldsymbol{\omega}_{0}\right) \\
q_{\Phi 3}\left(t-t_{0}, \boldsymbol{\omega}_{0}\right) \\
q_{\Phi 4}\left(t-t_{0}, \boldsymbol{\omega}_{0}\right)
\end{array}\right]=\mathbf{q}_{\Phi}\left(t-t_{0}, \boldsymbol{\omega}_{0}\right)=\mathbf{q}_{s}\left(t-t_{0}, \boldsymbol{\omega}_{0}\right) \otimes \mathbf{q}_{h}\left(t-t_{0}, \boldsymbol{\omega}_{0}\right)
$$

with

$$
\begin{aligned}
\mathbf{q}_{s}\left(t-t_{0}, \boldsymbol{\omega}_{0}\right)= & {\left[\begin{array}{c}
0 \\
0 \\
\sin \left\{0.5\left(t-t_{0}\right) \omega_{n b}\right\} \\
\cos \left\{0.5\left(t-t_{0}\right) \omega_{n b}\right\}
\end{array}\right] } \\
\mathbf{q}_{h}\left(t-t_{0}, \boldsymbol{\omega}_{0}\right)= & {\left[\begin{array}{c}
\left(\frac{I_{S C} \boldsymbol{\omega}_{0}}{\left\|I_{S C} \boldsymbol{\omega}_{0}\right\|}\right) \sin \left\{0.5\left(t-t_{0}\right) \omega_{n s}\right\} \\
\cos \left\{0.5\left(t-t_{0}\right) \boldsymbol{\omega}_{n s}\right\}
\end{array}\right] }
\end{aligned}
$$

These formulas assume that the nominal spin axis is the body $z$ axis and that $I_{s c}=\operatorname{diag}\left(I_{T}, I_{T}, I_{S}\right)$. The $z$-component of the spin rate is $\omega_{z}(t)=\omega_{z 0}$, a constant. The body-axes nutation rate is $\omega_{n b}=\left[1-\left(I_{S} / I_{T}\right)\right] \omega_{z 0}$, and the nutation rate with respect to inertial space is $\omega_{n s}=\left\|I_{s c} \boldsymbol{\omega}_{0}\right\| / I_{T}$. Thus, $I_{S}$ is the moment of inertia about the nominal spin axis, $I_{T}$ is the moment of inertia about the two transverse axes, $\mathbf{q}_{s}\left(t-t_{0}, \boldsymbol{\omega}_{0}\right)$ parameterizes a rotation about the nominal spin axis at the body-axes nutation rate, and $\mathbf{q}_{h}\left(t-t_{0}, \boldsymbol{\omega}_{0}\right)$ parameterizes a rotation about the angular momentum vector at the inertial nutation rate.

One possible strategy for developing an analytic solution to the restricted second problem is to equate the $\mathbf{q}_{\text {rot } 21}$ rotation quaternion of equation (14) with $\mathbf{q}_{\Phi}\left(t_{2}-t_{1}, \boldsymbol{\omega}_{1}\right)$ from equation (26), where $\boldsymbol{\omega}_{1}=\boldsymbol{\omega}\left(t_{1}\right)$. This equation might be used to solve for $\boldsymbol{\omega}_{1}$ or for $\mathbf{h}_{s c 1}=I_{s c} \boldsymbol{\omega}_{1}$ as a function of $\theta_{1}$ and $\theta_{2}$. The resulting solution could then be used to form $\mathbf{q}_{\Phi}\left(t_{3}-t_{1}, \boldsymbol{\omega}_{1}\right)$, and this rotation could be used to set up the three equations

$$
\mathbf{q}_{\alpha 3} \cos \left(\theta_{3}\right)+\mathbf{q}_{\beta 3} \sin \left(\theta_{3}\right)=\mathbf{q}_{\Phi}\left[t_{3}-t_{1}, \boldsymbol{\omega}_{1}\left(\theta_{1}, \theta_{2}\right)\right] \otimes\left[\mathbf{q}_{\alpha 1} \cos \left(\theta_{1}\right)+\mathbf{q}_{\beta 1} \sin \left(\theta_{1}\right)\right]
$$

in the three unknowns $\theta_{1}, \theta_{2}$, and $\theta_{3}$. This equation makes use of the formulas for $\mathbf{q}_{\alpha i}, \mathbf{q}_{\beta i}$, and $\mathbf{q}\left(t_{i}\right)$ that are given in equations (12a)-(13). Although equation (28) has four components, only three of them are independent due to the quaternion normalization constraint. If these three independent equations could be solved analytically for $\theta_{1}, \theta_{2}$, and $\theta_{3}$, then the result could be used to determine the solution to the restricted second problem $\left(\mathbf{q}_{0}, \boldsymbol{\omega}_{0}\right)$. 
An alternative solution strategy is to deal directly with six independent equations in six unknowns. Such an approach might work with the six unknowns $\theta_{1}, \theta_{2}$, and $\theta_{3}$ and the three elements of $\boldsymbol{\omega}_{2}=\boldsymbol{\omega}\left(t_{2}\right)$. One possible set of six independent equations is

$$
\begin{aligned}
& \mathbf{q}_{\alpha 3} \cos \left(\theta_{3}\right)+\mathbf{q}_{\beta 3} \sin \left(\theta_{3}\right)=\mathbf{q}_{\Phi}\left(t_{3}-t_{2}, \boldsymbol{\omega}_{2}\right) \otimes\left[\mathbf{q}_{\alpha 2} \cos \left(\theta_{2}\right)+\mathbf{q}_{\beta 2} \sin \left(\theta_{2}\right)\right] \\
& \mathbf{q}_{\alpha 1} \cos \left(\theta_{1}\right)+\mathbf{q}_{\beta 1} \sin \left(\theta_{1}\right)=\mathbf{q}_{\Phi}\left(t_{1}-t_{2}, \boldsymbol{\omega}_{2}\right) \otimes\left[\mathbf{q}_{\alpha 2} \cos \left(\theta_{2}\right)+\mathbf{q}_{\beta 2} \sin \left(\theta_{2}\right)\right]
\end{aligned}
$$

Actually, there are eight equations here, but only six are independent due to the normalization constraints of the quaternions.

It is not clear whether the above strategies and related methods will yield analytic solutions. Even if they do not, they might be useful for developing numerical solution procedures based on iterative equation solving techniques. It might be possible to manipulate equations like those above in order to reduce the number of unknowns that need to be determined numerically. Alternatively, these and related equations might enable the derivation of bounds on the space of the unknowns that delimit a finite region which is guaranteed to contain the global solution.

\section{Candidate Solution Strategies for the Two Generalized Wahba Problems}

This section proposes strategies that could be used for solving the two original generalized Wahba problems. An obvious strategy is to use an inner-analytic solution for $\mathbf{q}_{0}$ based on the $q$ method. This would be augmented by an outer optimization for either $\omega$ or $\boldsymbol{\omega}_{0}$. The outer optimization might proceed numerically. This section outlines how a Newton-like optimization could be performed on this outer optimization.

\section{Inner $\mathbf{q}_{0}$ and Outer $\omega$ or $\boldsymbol{\omega}_{0}$ Optimizations}

The performance metric of the first generalized Wahba problem, $\bar{p}_{a}\left(\mathbf{q}_{0}, \omega\right)=\mathbf{q}_{0}^{\mathrm{T}} K_{a}(\omega) \mathbf{q}_{0}$, suggests a possible solution algorithm that decomposes the problem into an inner optimization of $\mathbf{q}_{0}$ with an outer optimization of $\omega$ wrapped around it. For any given value of $\omega$, the inner optimization can compute the globally maximizing $\mathbf{q}_{0}$ by solving an eigenvalue problem, as in the original $q$ method of equations (5a) and (5b). The performance metric can then be reduced to $\tilde{p}_{a}(\omega)=\lambda_{\max }(\omega)$. The outer optimization must find the scalar $\omega$ that maximizes $\lambda_{\max }(\omega)$.

The performance metric of the second generalized Wahba problem, $\bar{p}_{b}\left(\mathbf{q}_{0}, \omega_{0}\right)=\mathbf{q}_{0}^{\mathrm{T}} K_{b}\left(\boldsymbol{\omega}_{0}\right) \mathbf{q}_{0}$, is also amenable to an inner/outer solution procedure. The inner problem determines the globally optimal $\mathbf{q}_{0}$ for any given initial attitude rate $\boldsymbol{\omega}_{0}$. The performance metric for the outer optimization reduces to $\tilde{p}_{b}\left(\boldsymbol{\omega}_{0}\right)=\lambda_{\max }\left(\boldsymbol{\omega}_{0}\right)$. This optimization is more complicated than the outer optimization of the first generalized Wahba problem because this performance metric is a function of three scalar unknowns rather than just one.

An important property of the inner/outer approach is that the corresponding $\mathbf{q}_{0}$ estimate is guaranteed to be the global optimum for the given estimate of $\omega$ or $\boldsymbol{\omega}_{0}$. If a global outer optimization can also be performed, then the solution will be the global optimal solution to the corresponding generalized Wahba problem.

Figure 1 plots the performance metric $\tilde{p}_{a}(\omega)=\lambda_{\max }(\omega)$ vs. $\omega$ for a typical outer optimization of the first problem. This metric has been generated from a truth-model simulation that uses eight vector measurements that are distributed over 1.37 spin 
periods; the truth spin period is $45.32 \mathrm{sec}$. The accuracies of the attitude measurements range from 1.3 to $2.2 \mathrm{deg}$. The figure shows that the global maximum of the outer problem clearly falls very near the truth spin rate, within about $0.00011 \mathrm{rad} / \mathrm{sec}$. The corresponding optimal estimate of $\mathbf{q}_{0}$ has a total attitude error of $0.85 \mathrm{deg}$ from the truth value of $\mathbf{q}_{0}$, with errors about the individual body axes ranging from $0.09 \mathrm{deg}$ to $0.72 \mathrm{deg}$. Thus, the global outer optimization, which has been performed by brute-force calculation of $\lambda_{\max }(\omega)$ on a very fine grid of $\omega$ values, yields accurate estimates for the attitude and the spin rate.

Figure 1 also shows two local maxima of the $\tilde{p}_{a}(\omega)$ performance metric that are not the global maximum. These occur at $\omega=0.019 \mathrm{rad} / \mathrm{sec}$ and at $\omega=0.255 \mathrm{rad} / \mathrm{sec}$. There may well be additional local maxima outside the range of $\omega$ values that have been considered. The possibility of multiple local maxima presents a challenge to any outer optimization algorithm that seeks to find the global maximum.

The sharp corner in the $\lambda_{\max }$ vs. $\omega$ curve of Fig. 1 indicates a discontinuous first derivative of the performance metric at $\omega=0.216 \mathrm{rad} / \mathrm{sec}$. This singularity is caused by a change in which eigenvalue of $K_{a}(\omega)$ constitutes the maximum. This change is illustrated by the grey curve, which plots the second largest eigenvalue of $K_{a}(\omega)$ vs. $\omega$. This grey curve intersects the black dash-dotted curve at the point where the latter curve has a discontinuous slope. The original maximum eigenvalue at lower $\omega$ values decreases with increases of $\omega$ while the second largest eigenvalue increases with $\omega$ until the two values cross. The possibility of such corners in the outer performance metric can cause a problem for gradient-based numerical optimization procedures.

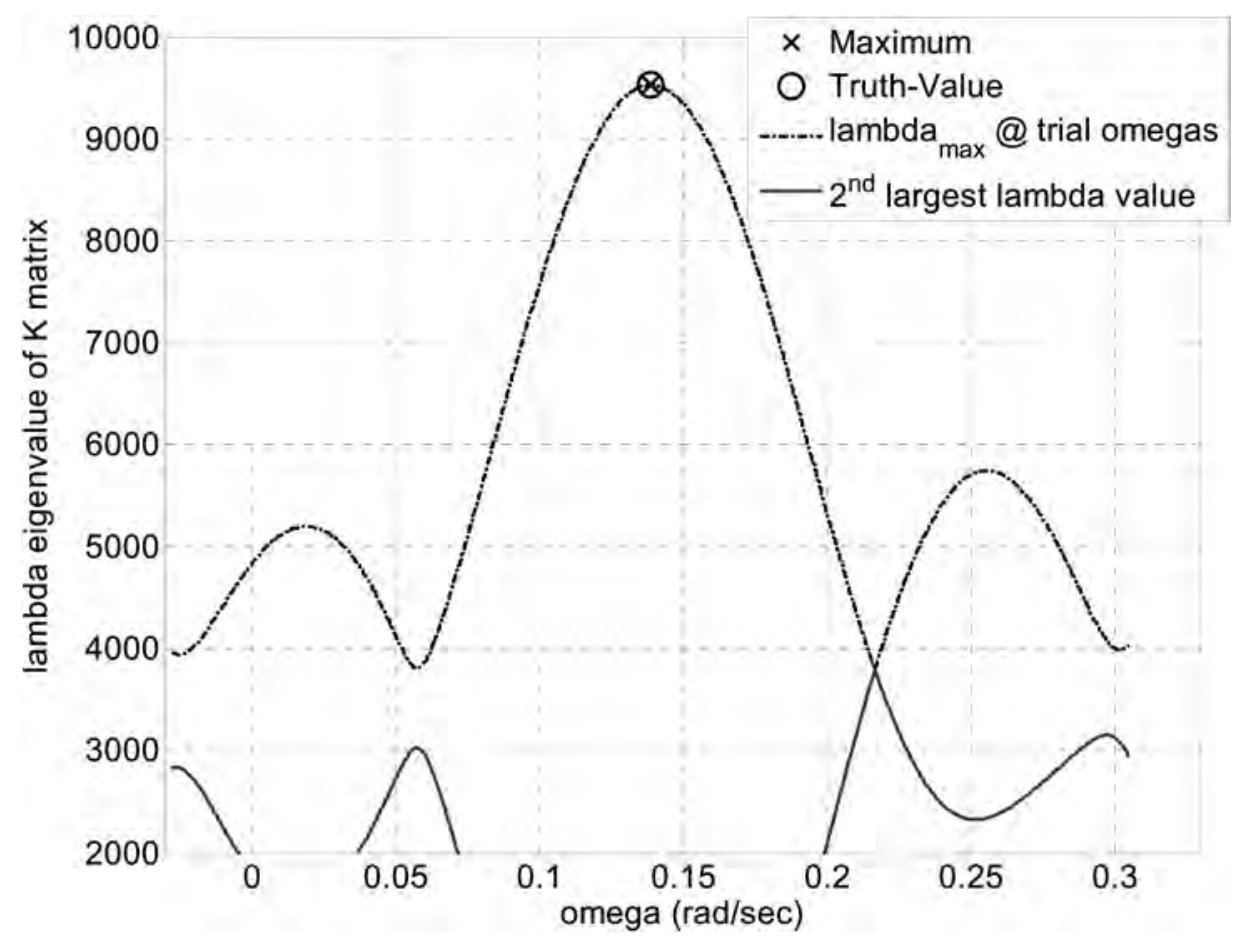

FIG. 1. $\lambda_{\max }$ vs. $\omega$ for the Outer $\omega$ Optimization of an Example First Generalized Wahba Problem. 


\section{Iterative Numerical Solution of the Outer $\omega$ or $\boldsymbol{\omega}_{0}$ Optimization}

The outer $\omega$ optimization for the first generalized Wahba problem and the outer $\boldsymbol{\omega}_{0}$ optimization for the second problem could be performed using an iterative nonlinear numerical optimization routine. A natural choice would be Newton's method.

Newton's method for nonlinear optimization computes solution increments by maximizing a local quadratic approximation of the performance metric [15]. In the outer $\omega$ optimization of the first problem, this quadratic cost function would be

$$
\Delta \tilde{p}_{a}(\Delta \omega)=\left.\frac{\partial \lambda_{\max }}{\partial \omega}\right|_{\omega_{g}} \Delta \omega+\left.\frac{1}{2} \frac{\partial^{2} \lambda_{\max }}{\partial \omega^{2}}\right|_{\omega_{g}} \Delta \omega^{2}
$$

where $\omega_{g}$ is the current best estimate of the spin rate as determined by the Newton algorithm and where the optimal $\Delta \omega$ would be added to $\omega_{g}$ in order to generate the next estimate. The Newton increment for the second problem would be the $\Delta \boldsymbol{\omega}_{0}$ value that maximized the quadratic performance metric

$$
\Delta \tilde{p}_{b}\left(\Delta \boldsymbol{\omega}_{0}\right)=\left.\frac{\partial \lambda_{\max }}{\partial \boldsymbol{\omega}_{0}}\right|_{\boldsymbol{\omega}_{0 g}} \Delta \boldsymbol{\omega}_{0}+\left.\frac{1}{2} \Delta \boldsymbol{\omega}_{0}^{\mathrm{T}} \frac{\partial^{2} \lambda_{\max }}{\partial \boldsymbol{\omega}_{0}^{2}}\right|_{\boldsymbol{\omega}_{0 g}} \Delta \boldsymbol{\omega}_{0}
$$

with $\boldsymbol{\omega}_{0 g}$ being the Newton algorithm's current best estimate of the initial angular velocity vector and with the optimal $\Delta \boldsymbol{\omega}_{0}$ being the algorithm's next increment to this estimate.

An equivalent method for determining the $\Delta \boldsymbol{\omega}_{0}$ vector that maximizes the equation (31) metric is to solve an approximate quadratic optimization problem, one like equations (22a) $-(22 \mathrm{c})$, but with the following modifications: the gradient vectors $\mathbf{g}_{q}$ and $\mathbf{g}_{\omega}$ and the Hessian matrices $H_{q q}, H_{q \omega}$, and $H_{\omega \omega}$ in equation (22b) must be computed at $\boldsymbol{\omega}_{0}=\boldsymbol{\omega}_{0 g}$ and at the $\mathbf{q}_{0 g}$ value that satisfies $K_{b}\left(\boldsymbol{\omega}_{0 g}\right) \mathbf{q}_{0 g}=\lambda_{g} \mathbf{q}_{0 g}$, with $\mathbf{q}_{0 g}^{\mathrm{T}} \mathbf{q}_{0 g}=1$ and with $\lambda_{g}$ being the maximum eigenvalue of $K_{b}\left(\boldsymbol{\omega}_{0 g}\right)$. The term $\lambda_{\text {opt }}$ in equation (22b) must be replaced by $\lambda_{g}$. Also, the term $\mathbf{q}_{\text {oopt }}^{\mathrm{T}}$ in equation (22c) must be replaced by $\mathbf{q}_{0 g}^{\mathrm{T}}$.

A similar method can be developed for determining $\Delta \omega$ for the outer numerical optimization of the first generalized Wahba problem. This method works by deriving a local quadratic approximation of the first problem that is similar to equations (22a)-(22c), except that the needed matrix derivatives $\mathrm{d} K_{a} / \mathrm{d} \omega$ and $\mathrm{d}^{2} K_{a} / \mathrm{d} \omega^{2}$ are calculated using simpler operations than those in equations (A3a)-(A3c) and (A4a)-(A4c) of the Appendix.

Any gradient-based outer optimization would need to be augmented with strategies for dealing with the two possible difficulties that have been illustrated in Fig. 1. Gradient-based algorithms can only be guaranteed to find a local maximum, and this guarantee holds true only if additional guarding operations are added to the computation of the increment $\Delta \omega$ or $\Delta \omega_{0}$. An additional outer loop would have to be wrapped around a gradient-based technique in order to seek multiple local maxima in hopes of finding the global maximum. Any gradient-based algorithm would also need to be augmented to deal with the possibility of one or more isolated discontinuities of the first derivative of the performance metric, as illustrated in Fig. 1 at $\omega=0.216 \mathrm{rad} / \mathrm{sec}$.

\section{Summary and Conclusions}

This paper has posed two generalized versions of Wahba's attitude determination problem. The generalized problems seek to estimate the attitude and the angular rate 
of a spinning spacecraft based on a time series of vector attitude observations coupled with a dynamic model of the spacecraft spin. One generalized problem presumes a known spin axis and solves for the unknown constant spin rate about this axis along with the initial attitude quaternion. It is applicable to a spin-stabilized spacecraft that uses a nutation damper. The other generalized problem presumes a known inertia matrix and torque-free motion and solves for the initial quaternion and the initial rotation rate vector. This second problem is applicable to a spinning spacecraft that is undergoing significant rigid-body nutations. These two problems take similar mathematical forms: a Wahba problem with a $K$ matrix that depends on unknown rate parameters.

This paper has posed restricted forms of the generalized Wahba problems that limit the number of vector measurements to the minimum required for observability. That number is two for the first problem and three for the second problem. An analytic solution to the restricted form of the first problem has been presented. The restricted form of the second problem has been shown to be observable, and solution strategies for this problem have been suggested.

A semi-analytic solution strategy has been proposed for the unrestricted forms of the two generalized Wahba problems. This approach solves an inner quaternion optimization using the closed-form $q$ method, and it employs an iterative numerical optimization to solve the outer problem for the attitude rate parameters. This outer optimization must deal with the possibilities that its performance metric will have multiple local optima and discontinuities in its first derivative.

The new generalized Wahba problems and the associated solution strategies will be useful for spin-stabilized spacecraft missions that carry vector attitude sensors but no rate gyros. A global solution to one of the generalized Wahba problems could provide a reliable initial state estimate for an extended Kalman filter, or it could be used as a batch algorithm for attitude and angular rate determination over finite windows of time.

\section{References}

[1] PSIAKI, M. L., KLATT, E. M., KINTNER, P. M., Jr., and POWELL, S. P. “Attitude Estimation for a Flexible Spacecraft in an Unstable Spin," Journal of Guidance, Control, and Dynamics, Vol. 25, No. 1, Jan.-Feb. 2002, pp. 88-95.

[2] MARKLEY, F. L. and SEDLAK, J.E. "Kalman Filter for Spinning Spacecraft Attitude Estimation," Journal of Guidance, Control, and Dynamics, Vol. 31, No. 6, Nov.-Dec. 2008, pp. $1750-1760$.

[3] CRASSIDIS, J. L., MARKLEY, F.L., and CHENG, Y. "Survey of Nonlinear Attitude Estimation Methods," Journal of Guidance, Control, and Dynamics, Vol. 30, No. 1, Jan.-Feb. 2007, pp. 12-28.

[4] PSIAKI, M. L. "Backward-Smoothing Extended Kalman Filter," Journal of Guidance, Control, and Dynamics, Vol. 28, No. 5, Sept.-Oct. 2005, pp. 885-894.

[5] WAHBA, G. "A Least Squares Estimate of Satellite Attitude," SIAM Review, Vol. 7, No. 3, 1965 , p. 409.

[6] WERTZ, J. R., ed. Spacecraft Attitude Determination and Control, D. Reidel Pub. Co., Boston, 1978, pp. 426-428, 487-494, 511-512, 521-531, 758-759.

[7] MARKLEY, F. L. "Attitude Determination and Parameter Estimation Using Vector Observations: Theory," The Journal of the Astronautical Sciences, Vol. 37, No. 1, 1989, pp. 41-58.

[8] MARKLEY, F. L. "Attitude Determination and Parameter Estimation Using Vector Observations: Application," The Journal of the Astronautical Sciences, Vol. 39, No. 3, 1991, pp. 367-381.

[9] SHUSTER, M. D. "A Simple Kalman Filter and Smoother for Spacecraft Attitude," The Journal of the Astronautical Sciences, Vol. 37, No. 1, 1989, pp. 89-106.

[10] BAR-ITZHACK, I. Y. "REQUEST: A Recursive QUEST Algorithm for Sequential Attitude Determination," Journal of Guidance, Control, and Dynamics, Vol. 19, No. 5, Sept.-Oct. 1996, pp. $1034-1038$. 
[11] PSIAKI, M.L. "Attitude-Determination Filtering via Extended Quaternion Estimation," Journal of Guidance, Control, and Dynamics, Vol. 23, No. 2, March-April 2000, pp. 206-214.

[12] SHUSTER, M.D. "The Generalized Wahba Problem," The Journal of the Astronautical Sciences, Vol. 54, No. 2, 2006, pp. 245-259.

[13] DEPRIT, A. and ELIPE, A. "Complete Reduction of the Euler-Poinsot Problem," The Journal of the Astronautical Sciences, Vol. 41, No. 4, Oct.-Dec. 1993, pp. 603-628.

[14] REYNOLDS, R. G. "Quaternion Parameterization and a Simple Algorithm for Global Attitude Estimation," Journal of Guidance, Control, and Dynamics, Vol. 21, No. 4, July-Aug. 1998, pp. 669-671.

[15] GILL, P.E., MURRAY, W., and WRIGHT, M.H. Practical Optimization, Academic Press, New York, 1981, pp. 105-115.

\section{Appendix: Partial Derivatives for the Second Order Approximation of the Second Problem}

The gradients of the equation (9b) metric $\bar{p}_{b}\left(\mathbf{q}_{0}, \boldsymbol{\omega}_{0}\right)$ with respect to $\mathbf{q}_{0}$ and $\boldsymbol{\omega}_{0}$ are the vectors

$$
\begin{aligned}
& \mathbf{g}_{q}=\left[\left.\frac{\partial \bar{p}_{b}}{\partial \mathbf{q}_{0}}\right|_{\left(\mathbf{q}_{0 \text { opt }}, \boldsymbol{\omega}_{0 \text { opt }}\right.}\right]^{\mathrm{T}}=2 K_{b}\left(\boldsymbol{\omega}_{0 \text { opt }}\right) \mathbf{q}_{\text {oopt }} \\
& \mathbf{g}_{\omega}=\left[\left.\frac{\partial \bar{p}_{b}}{\partial \boldsymbol{\omega}_{0}}\right|_{\left(\mathbf{q}_{0 \text { opt }}, \omega_{0 \text { opt }}\right)}\right]^{\mathrm{T}}=\left[\begin{array}{l}
\left.\mathbf{q}_{\text {oopt }}^{\mathrm{T}} \frac{\partial K_{b}}{\partial \omega_{x 0}}\right|_{\omega_{0 \text { opt }}} \mathbf{q}_{\text {oopt }} \\
\left.\mathbf{q}_{\text {oopt }}^{\mathrm{T}} \frac{\partial K_{b}}{\partial \omega_{00}}\right|_{\omega_{0 \text { opt }}} ^{\mathbf{q}_{\text {oopt }}} \\
\left.\mathbf{q}_{\text {oopt }}^{\mathrm{T}} \frac{\partial K_{b}}{\partial \omega_{z 0}}\right|_{\boldsymbol{\omega}_{0 \text { opt }}} ^{\mathbf{q}_{\text {oopt }}}
\end{array}\right]
\end{aligned}
$$

The corresponding second derivative Hessian matrices are

$$
\begin{aligned}
& H_{q q}=\left.\frac{\partial^{2} \bar{p}_{b}}{\partial \mathbf{q}_{0}^{2}}\right|_{\left(\mathbf{q}_{0 o p t}, \omega_{0 o p t}\right)}=2 K_{b}\left(\boldsymbol{\omega}_{0 \text { opt }}\right) \\
& H_{q \omega}=\left.\frac{\partial^{2} \bar{p}_{b}}{\partial \mathbf{q}_{0} \partial \boldsymbol{\omega}_{0}}\right|_{\left(\mathbf{q}_{0 o p t}, \omega_{0 o p t}\right)} \\
& =2\left[\left(\left.\frac{\partial K_{b}}{\partial \omega_{x 0}}\right|_{\omega_{0 o p t}} \mathbf{q}_{0 \text { opt }}\right),\left(\left.\frac{\partial K_{b}}{\partial \omega_{y 0}}\right|_{\omega_{0 o p t}} \mathbf{q}_{0 o p t}\right),\left(\left.\frac{\partial K_{b}}{\partial \omega_{z 0}}\right|_{\omega_{0 o p t}} \mathbf{q}_{0 o p t}\right)\right] \\
& H_{\omega \omega}=\left.\frac{\partial^{2} \bar{p}_{b}}{\partial \boldsymbol{\omega}_{0}^{2}}\right|_{\left(\mathbf{q}_{0 o p t}, \omega_{0 o p t}\right)}
\end{aligned}
$$

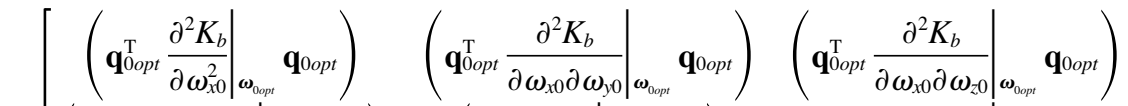

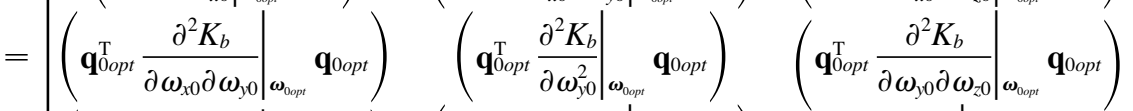

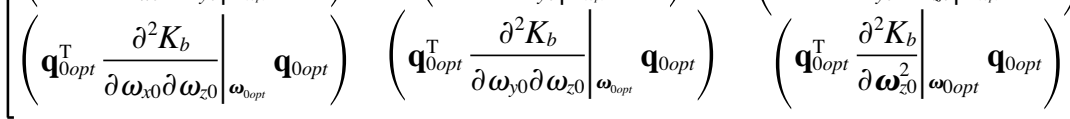

The first and second partial derivatives with respect to $\boldsymbol{\omega}_{0}$ of the symmetric $4 \times 4$ matrix $K_{b}$ can be computed by differentiating equations (10a)-(11) appropriately. The needed first derivatives of these equations are 


$$
\begin{aligned}
\frac{\mathrm{d}}{\mathrm{d} t} \frac{\partial \boldsymbol{\omega}(t)}{\partial \omega_{j 0}}= & -I_{s c}^{-1}\left\{\frac{\partial \boldsymbol{\omega}(t)}{\partial \omega_{j 0}} \times\left[I_{s c} \boldsymbol{\omega}(t)\right]+\boldsymbol{\omega}(t) \times\left[I_{S C} \frac{\partial \boldsymbol{\omega}(t)}{\partial \omega_{j 0}}\right]\right\} \\
\frac{\mathrm{d}}{\mathrm{d} t} \frac{\partial \Phi\left(t, t_{0}\right)}{\partial \omega_{j 0}}= & \frac{1}{2}\left[\begin{array}{cccc}
0 & \frac{\partial \omega_{z}(t)}{\partial \omega_{j 0}} & -\frac{\partial \omega_{y}(t)}{\partial \omega_{j 0}} & \frac{\partial \omega_{x}(t)}{\partial \omega_{j 0}} \\
-\frac{\partial \omega_{z}(t)}{\partial \omega_{j 0}} & 0 & \frac{\partial \omega_{x}(t)}{\partial \omega_{j 0}} & \frac{\partial \omega_{y}(t)}{\partial \omega_{j 0}} \\
\frac{\partial \omega_{y}(t)}{\partial \omega_{j 0}} & -\frac{\partial \omega_{x}(t)}{\partial \omega_{j 0}} & 0 & \frac{\partial \omega_{z}(t)}{\partial \omega_{j 0}} \\
-\frac{\partial \omega_{x}(t)}{\partial \omega_{j 0}} & -\frac{\partial \omega_{y}(t)}{\partial \omega_{j 0}} & -\frac{\partial \omega_{z}(t)}{\partial \omega_{j 0}} & 0
\end{array}\right] \Phi\left(t, t_{0}\right) \\
& +\frac{1}{2}\left[\begin{array}{cccc}
0 & \omega_{z}(t) & -\omega_{y}(t) & \omega_{x}(t) \\
-\omega_{z}(t) & 0 & \omega_{x}(t) & \omega_{y}(t) \\
\omega_{y}(t) & -\omega_{x}(t) & 0 & \omega_{z}(t) \\
-\omega_{x}(t) & -\omega_{y}(t) & -\omega_{z}(t) & 0
\end{array}\right] \\
\frac{\partial K_{b}}{\partial \omega_{j 0}}= & \sum_{i=1}^{m}\left[\begin{array}{cccc}
\frac{\partial \Phi^{\mathrm{T}}\left(t_{i}, t_{0}\right)}{\partial \omega_{j 0}} & K_{i} \Phi\left(t_{i}, t_{0}\right) & +\Phi^{\mathrm{T}}\left(t_{i}, t_{0}\right) K_{i} \frac{\partial \Phi\left(t_{i}, t_{0}\right)}{\partial \omega_{j 0}}
\end{array}\right]
\end{aligned}
$$

for $j=x, y$, or $z$. The initial conditions for the joint vector/matrix initial value problem in equations (A3a) and (A3b) are $\partial \boldsymbol{\omega}\left(t_{0}\right) / \partial \omega_{x 0}=[1 ; 0 ; 0]$, $\partial \boldsymbol{\omega}\left(t_{0}\right) / \partial \omega_{y 0}=[0 ; 1 ; 0]$, or $\partial \boldsymbol{\omega}\left(t_{0}\right) / \partial \omega_{z 0}=[0 ; 0 ; 1]$, and $\partial \Phi\left(t_{0}, t_{0}\right) / \partial \omega_{j 0}=0_{4 \times 4}$. The second derivative computation takes the form

$$
\begin{aligned}
& \frac{\mathrm{d}}{\mathrm{d} t} \frac{\partial^{2} \boldsymbol{\omega}(t)}{\partial \omega_{j 0} \partial \omega_{k 0}}=-I_{S C}^{-1}\left\{\frac{\partial^{2} \boldsymbol{\omega}(t)}{\partial \boldsymbol{\omega}_{j 0} \partial \boldsymbol{\omega}_{k 0}} \times\left[I_{s c} \boldsymbol{\omega}(t)\right]+\frac{\partial \boldsymbol{\omega}(t)}{\partial \boldsymbol{\omega}_{j 0}} \times\left[I_{S C} \frac{\partial \boldsymbol{\omega}(t)}{\partial \boldsymbol{\omega}_{k 0}}\right]\right. \\
& \left.+\frac{\partial \boldsymbol{\omega}(t)}{\partial \omega_{k 0}} \times\left[I_{S C} \frac{\partial \boldsymbol{\omega}(t)}{\partial \omega_{j 0}}\right]+\boldsymbol{\omega}(t) \times\left[I_{S C} \frac{\partial^{2} \boldsymbol{\omega}(t)}{\partial \omega_{j 0} \partial \omega_{k 0}}\right]\right\} \\
& \left.\frac{\mathrm{d}}{\mathrm{d} t} \frac{\partial^{2} \Phi\left(t, t_{0}\right)}{\partial \omega_{j 0} \partial \omega_{k 0}}=\frac{1}{2}\left[\begin{array}{cccc}
0 & \frac{\partial^{2} \omega_{z}(t)}{\partial \omega_{j 0} \partial \omega_{k 0}} & -\frac{\partial^{2} \omega_{y}(t)}{\partial \omega_{j 0} \partial \omega_{k 0}} & \frac{\partial^{2} \omega_{x}(t)}{\partial \omega_{j 0} \partial \omega_{k 0}} \\
-\frac{\partial^{2} \omega_{z}(t)}{\partial \omega_{j 0} \partial \omega_{k 0}} & 0 & \frac{\partial^{2} \omega_{x}(t)}{\partial \omega_{j 0} \partial \omega_{k 0}} & \frac{\partial^{2} \omega_{y}(t)}{\partial \omega_{j 0} \partial \omega_{k 0}} \\
\frac{\partial^{2} \omega_{y}(t)}{\partial \omega_{j 0} \partial \omega_{k 0}} & -\frac{\partial^{2} \omega_{x}(t)}{\partial \omega_{j 0} \partial \omega_{k 0}} & 0 & \frac{\partial^{2} \omega_{z}(t)}{\partial \omega_{j 0} \partial \omega_{k 0}} \\
-\frac{\partial^{2} \omega_{x}(t)}{\partial \omega_{j 0} \partial \omega_{k 0}} & -\frac{\partial^{2} \omega_{y}(t)}{\partial \omega_{j 0} \partial \omega_{k 0}} & -\frac{\partial^{2} \omega_{z}(t)}{\partial \omega_{j 0} \partial \omega_{k 0}} & 0
\end{array}\right]\right]_{\left(t, t_{0}\right)} \\
& +\frac{1}{2}\left[\begin{array}{cccc}
0 & \frac{\partial \omega_{z}(t)}{\partial \omega_{j 0}} & -\frac{\partial \omega_{y}(t)}{\partial \omega_{j 0}} & \frac{\partial \omega_{x}(t)}{\partial \omega_{j 0}} \\
-\frac{\partial \omega_{z}(t)}{\partial \omega_{j 0}} & 0 & \frac{\partial \omega_{x}(t)}{\partial \omega_{j 0}} & \frac{\partial \omega_{y}(t)}{\partial \omega_{j 0}} \\
\frac{\partial \omega_{y}(t)}{\partial \omega_{j 0}} & -\frac{\partial \omega_{x}(t)}{\partial \omega_{j 0}} & 0 & \frac{\partial \omega_{z}(t)}{\partial \omega_{j 0}} \\
-\frac{\partial \omega_{x}(t)}{\partial \omega_{j 0}} & -\frac{\partial \omega_{y}(t)}{\partial \omega_{j 0}} & -\frac{\partial \omega_{z}(t)}{\partial \omega_{j 0}} & 0
\end{array}\right] \frac{\partial \Phi\left(t, t_{0}\right)}{\partial \omega_{k 0}}
\end{aligned}
$$




$$
\begin{aligned}
& +\frac{1}{2}\left[\begin{array}{cccc}
0 & \frac{\partial \omega_{z}(t)}{\partial \omega_{k 0}} & -\frac{\partial \omega_{y}(t)}{\partial \omega_{k 0}} & \frac{\partial \omega_{x}(t)}{\partial \omega_{k 0}} \\
-\frac{\partial \omega_{z}(t)}{\partial \omega_{k 0}} & 0 & \frac{\partial \omega_{x}(t)}{\partial \omega_{k 0}} & \frac{\partial \omega_{y}(t)}{\partial \omega_{k 0}} \\
\frac{\partial \omega_{y}(t)}{\partial \omega_{k 0}} & -\frac{\partial \omega_{x}(t)}{\partial \omega_{k 0}} & 0 & \frac{\partial \omega_{z}(t)}{\partial \omega_{k 0}} \\
-\frac{\partial \omega_{x}(t)}{\partial \omega_{k 0}} & -\frac{\partial \omega_{y}(t)}{\partial \omega_{k 0}} & -\frac{\partial \omega_{z}(t)}{\partial \omega_{k 0}} & 0
\end{array}\right] \frac{\partial \Phi\left(t, t_{0}\right)}{\partial \omega_{j 0}} \\
& +\frac{1}{2}\left[\begin{array}{cccc}
0 & \omega_{z}(t) & -\omega_{y}(t) & \omega_{x}(t) \\
-\omega_{z}(t) & 0 & \omega_{x}(t) & \omega_{y}(t) \\
\omega_{y}(t) & -\omega_{x}(t) & 0 & \omega_{z}(t) \\
-\omega_{x}(t) & -\omega_{y}(t) & -\omega_{z}(t) & 0
\end{array}\right] \frac{\partial^{2} \Phi\left(t, t_{0}\right)}{\partial \omega_{j 0} \partial \omega_{k 0}} \\
& \frac{\partial^{2} K_{b}}{\partial \omega_{j 0} \partial \omega_{k 0}}=\sum_{i=1}^{m}\left[\frac{\partial^{2} \Phi^{\mathrm{T}}\left(t_{i}, t_{0}\right)}{\partial \omega_{j 0} \partial \omega_{k 0}} K_{i} \Phi\left(t_{i}, t_{0}\right)+\frac{\partial \Phi^{\mathrm{T}}\left(t_{i}, t_{0}\right)}{\partial \omega_{j 0}} K_{i} \frac{\partial \Phi\left(t_{i}, t_{0}\right)}{\partial \omega_{k 0}}\right. \\
& \left.+\frac{\partial \Phi^{\mathrm{T}}\left(t_{i}, t_{0}\right)}{\partial \omega_{k 0}} K_{i} \frac{\partial \Phi\left(t_{i}, t_{0}\right)}{\partial \omega_{j 0}}+\Phi^{\mathrm{T}}\left(t_{i}, t_{0}\right) K_{i} \frac{\partial^{2} \Phi\left(t_{i}, t_{0}\right)}{\partial \omega_{j 0} \partial \omega_{k 0}}\right]
\end{aligned}
$$

for $j=x, y$, or $z$ and $k=x, y$, or $z$. The initial conditions for the joint vector/matrix initial value problem in equations (A4a) and (A4b) are $\partial^{2} \boldsymbol{\omega}\left(t_{0}\right) / \partial \omega_{j 0} \partial \omega_{k 0}=0_{3 \times 1}$ and $\partial^{2} \Phi\left(t_{0}, t_{0}\right) / \partial \omega_{j 0} \partial \omega_{k 0}=0_{4 \times 4}$. It is usually advantageous to perform numerical integration of the initial value problems in equations (10a) (10b), (A3a) (A3b), (A4a), and (A4b) simultaneously because solutions from some of these equations are needed in others at intermediate times. 